\title{
Amplifying hydrogen: neutron diffraction using Dynamic Nuclear Polarization
}

\author{
D.A. Myles ${ }^{1}$, J. Pierce ${ }^{1}$, M.J. Cuneo ${ }^{2}$, K. Herwig ${ }^{1}$, F. Meilleur ${ }^{1,3}$, J. Zhao ${ }^{4}$ \\ ${ }^{1}$ Neutron Science Directorate, Oak Ridge National Laboratory, Oak Ridge, TN 37831., \\ ${ }^{2}$ Depart. of Structural Biology, St. Jude Children's Research Hospital, Memphis, TN 38105. \\ ${ }^{3}$ Depart. of Molecular \& Structural Biochemistry, North Carolina State University, Raleigh, NC 27695 \\ ${ }^{4}$ Beijing National Laboratory for Condensed Matter Physics, Institute of Physics, Chinese Academy of Sciences, \\ Beijing 100190, China \\ mylesda@ornl.gov
}

Harnessing the spin dependence of the neutron scattering cross section for hydrogen, Dynamic Nuclear Polarization (DNP) is a potentially powerful technique for neutron diffraction measurements, especially for biological systems. Polarizing the neutron beam and aligning the proton spins in a polarized sample modulates and tunes the coherent and incoherent neutron scattering cross-sections of hydrogen [1], in ideal cases maximizing the scattering from - and visibility of - hydrogen atoms in the sample while simultaneously minimizing the incoherent background to zero (see Figure 1).

ORNL has developed a prototype system for the purpose of performing proof-of-concept Neutron Macromolecular Crystallography measurements which highlight the potential of DNP [2]. We will describe DNP concepts, experimental design, labelling strategies and the most recent results, as well as considering future prospects for data collection and analysis that these techniques enable.

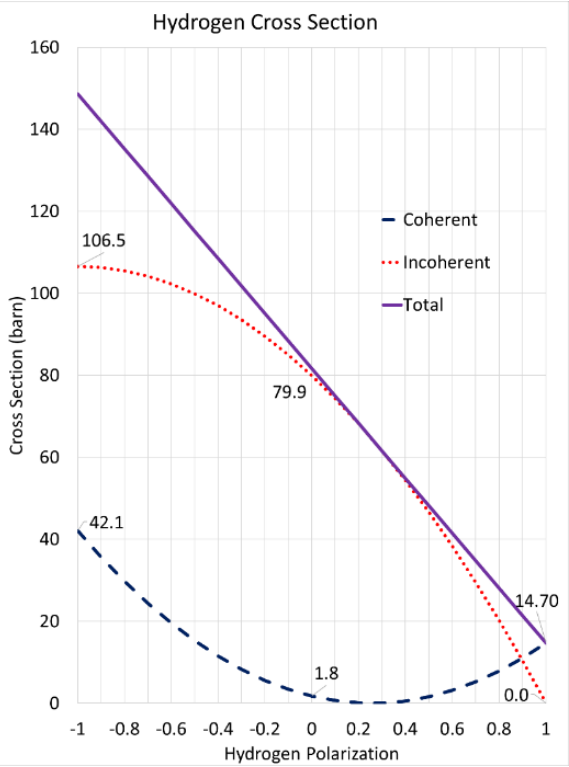

Figure 1. Coherent, incoherent and total scattering cross section of hydrogen as a function of the proton polarization for fully polarized neutrons.

[1] Stuhrmann, H.B., (2004), Reports on Progress in Physics. 67, 1073-1115.

[2] Pierce, J., Cuneo, M. J., Jennings, A., Li, L., Meilleur, F., Zhao, J. \& Myles D. A. A. (2020), Methods in Enzymology, 634, 153-175

Keywords: Neutron diffraction; protein; hydrogen; dynamic nuclear polarization (DNP) 